EPJ Web of Conferences 41, 05005 (2013)

DOI: $10.1051 /$ epjconf/20134105005

(C) Owned by the authors, published by EDP Sciences, 2013

\title{
Monitoring of the ultrafast vibrational kinetic during formation of photo-induced linkage isomers in $\mathrm{Na}_{2}\left[\mathrm{Fe}(\mathrm{CN})_{5} \mathrm{NO}\right] \cdot 2 \mathrm{H}_{2} \mathrm{O}$ single crystal
}

\author{
G. Galle ${ }^{1}$, M. Nicoul ${ }^{2}$, Th. Woike ${ }^{3}$, D. Schaniel ${ }^{4}$ and E. Freysz ${ }^{1}$ \\ ${ }^{1}$ University of Bordeaux, CNRS-UMR 5798, LOMA, 351 cours de la Libération, Talence 33405, \\ France \\ ${ }^{2}$ Physikalisches Institut, Universität zu Köln, Zülpicherstr. 77, 50937 Köln, Germany \\ ${ }^{3}$ Institut für Strukturphysik, TU-Dresden, Zellescherweg 16, Dresden, Germany \\ ${ }^{4}$ CRM2, Institut Jean Barriol, Nancy Université, 54506 Vandoeuvre-les-Nancy, France
}

\begin{abstract}
A femtosecond visible pump - infrared probe time resolved absorption experiment makes it possible to reveal the ultrafast vibrationnal kinetic associated to formation of light-induced linkage isomers in $\mathrm{Na}_{2}\left[\mathrm{Fe}(\mathrm{CN})_{5} \mathrm{NO}\right] 2 \mathrm{H}_{2} \mathrm{O}$ (SNP) single crystals.
\end{abstract}

Time-resolved spectroscopy on a femtosecond scale makes it possible to observe and to record photochemical processes [1-2]. The ultrafast study of electronic, vibrational and structural changes during light-induced isomerization reveals the correlation between the changes of the electron density and the structural response of matter. Consider an electronic transition that excites a molecule from a (bonding) ground state to an (reactive) excited state that is the starting point for, e.g., the rotation of a ligand in a molecule. According to the Born-Oppenheimer approximation the direct electronic excitation in the sub-femtosecond range is followed by a slower nuclear response in the fs-ps range. The nuclear motion (e.g. rotation) of the ligand starts in a highly excited state and in the absence of luminescence will end in highly excited vibrational-rotational states of the novel geometry. The excess energy will be dissipated during the thermalization of this highly excited vibrational-rotational state towards its ground state. A typical example for such ultrafast photochemical processes is the photo-induced linkage isomerism of the nitrosyl ligand in coordination complexes [3]. Here we study the prototypic case of $\left[\mathrm{Fe}(\mathrm{CN})_{5} \mathrm{NO}\right]^{2-}$ anion. As shown in Figure 1 the ground state (GS) is characterized by a linear Fe-N-O coordination. The irradiation with light in the blue-green spectral range (e.g. $\lambda \sim 500 \mathrm{~nm}$ ) induces a charge-transfer transition. Thereby the system changes symmetry from a ${ }^{1} \mathrm{~A}_{1}$ state to a ${ }^{1} \mathrm{E}$ doubly degenerate state. As a consequence the doubly degenerate deformational mode $\delta(\mathrm{Fe}-\mathrm{N}-\mathrm{O})$ can induce a rotation of the NO ligand. The rotation of about $90^{\circ}$ yields the side-on configuration of $\mathrm{Fe}<{ }^{\mathrm{N}}$ (metastable state MS2) while a rotation of $180^{\circ}$ results in the isonitrosyl configuration Fe-O-N (metastable state MS1). The transition from the excited ground state ${ }^{1} \mathrm{E}$ towards MS2 occurs radiationless in about $300 \pm 30 \mathrm{fs}$ [3]. In the case of the NO ligand the structure of GS and the metastable states MS1 and MS2 is known from X-ray and neutron diffraction measurements at low temperatures in the static regime $[4,5,6]$. Moreover, the GS, MS1, and MS2 have clearly distinguished $v(\mathrm{NO})$ vibration frequency centered at $1961 \mathrm{~cm}^{-1}(5100 \mathrm{~nm}), 1831 \mathrm{~cm}^{-1}(5460 \mathrm{~nm})$ and $1631 \mathrm{~cm}^{-1}(6130 \mathrm{~nm})$ respectively [7]. Hence optical

This is an Open Access article distributed under the terms of the Creative Commons Attribution License 2.0, which permits unrestricted use, distribution, and reproduction in any medium, provided the original work is properly cited. 
pump and infrared probe time resolved absorption spectroscopy offers to the possibility to monitor the vibrational kinetic during photo-induced isomerization and makes an unambiguous identification of the corresponding relaxation pathways possible.

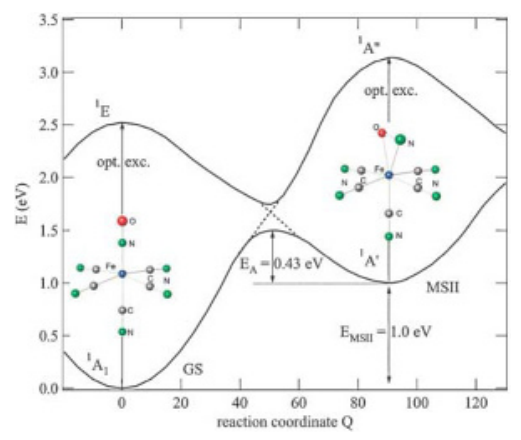

Fig. 1: Potential scheme for the formation of the side-on bound isomer MS2 in $\left[\mathrm{Fe}(\mathrm{CN})_{5} \mathrm{NO}\right]^{2}$

Our experimental set-up is based on a $1 \mathrm{kHz}$ Ti:Sapphire regenerative amplifier system producing $50 \mathrm{fs}, 4.5 \mathrm{~mJ}$ laser pulses centered at $800 \mathrm{~nm}$ (Coherent Legend USP + Legend Duo Elite). The output of the laser system was split in two $1 \mathrm{~mJ}$ laser pulses which pumped two Optical Parametric Amplifiers (OPA, Model TOPAS from Light Conversion). The pump beam generated by the first OPA centered at $\lambda \sim 500 \mathrm{~nm}$ and of energy of about $9 \mu \mathrm{J}$ was focused in the sample. The second OPA was equipped with a Degenerate Frequency Generation (DFG) module for the generation in the spectral range in between $2500 \mathrm{~nm}-10.000 \mathrm{~nm}$. The output energy is at maximum about $4 \mu \mathrm{J}$ in the $4000 \mathrm{~nm}\left(2500 \mathrm{~cm}^{-1}\right)$ to $6000 \mathrm{~nm}\left(1666 \mathrm{~cm}^{-1}\right)$ spectral range. The probe pulse energy used in our experiment was at least ten times lower than the pump pulse energy. The pump and probe pulses have duration of $\sim 100 \pm 20 \mathrm{fs}$ and $200 \pm 50 \mathrm{fs}$ respectively. The used sample is a 270 $\mu \mathrm{m}$ thick single crystals of $\mathrm{Na}_{2}\left[\mathrm{Fe}(\mathrm{CN})_{5} \mathrm{NO}^{2} 2 \mathrm{H}_{2} \mathrm{O}\right.$. It is orthorhombic (Pnnm) and was b-cut. We set the pump-polarization parallel c-axis while the probe polarization is parallel to a axis. All measurements were performed at room temperature.
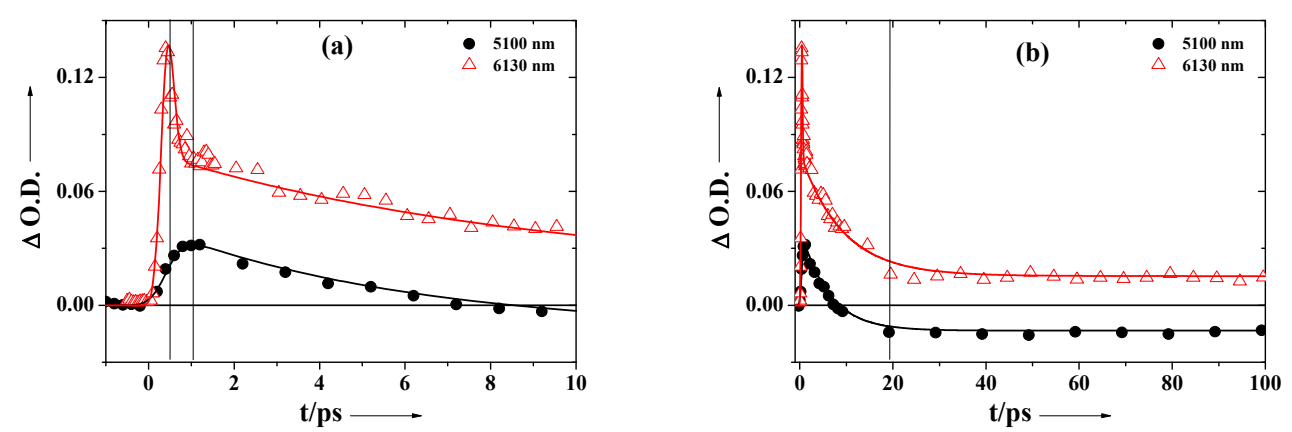

Fig. 2: Transient change of the optical density of the sample recorded for $1961 \mathrm{~cm}^{-1}(\bullet)$ and $1631 \mathrm{~cm}^{-1}(\Delta)$ as a function of the pump-probe delay. The solid lines are fits of the experimental data.

Figure 2 shows the temporal evolution of the optical density of the sample measured for the probe pulses centered along the $v(\mathrm{NO})$-vibration of GS $\left(1961 \mathrm{~cm}^{-1}\right)$ and MS2 $\left(1631 \mathrm{~cm}^{-1}\right)$ states after 
pump excitation. In all cases, the absorption increases during $2300 \mathrm{fs}$, that is about the pump-probe temporal overlap. The increase of the $v(\mathrm{NO})$-vibrations at $1631 \mathrm{~cm}^{-1}$ indicates the destabilization linear Fe-N-O arrangement in the ${ }^{1} \mathrm{E}$ excited state. The increase of the $v(\mathrm{NO})$-vibrations at $1961 \mathrm{~cm}^{-1}$ also reveals the higher cross section absorption of this vibration in this state. In good agreement with previous work, the ${ }^{1} \mathrm{E}$ excited state is found to last $\sim 250 \pm 30 \mathrm{fs}$. This accounts for the rapid decrease of the recorded absorption at $1631 \mathrm{~cm}^{-1}$ (MS2) as well as the increase of the absorption at $1961 \mathrm{~cm}^{-1}$ (GS). The latter increase is due to the fact that $95 \%$ of the photo-excited complexes relax back to the fundamental state where they are lived in a highly vibrationaly excited state [3]. Then, they relax vibrationaly towards the minimum of the potential well with a constant time of $5.8 \pm 0.4 \mathrm{ps}$. About $5 \%$ of the N-O ligand of the photo-excited complexes rotates by $90^{\circ}$. Once in the MS2 potential well, they are in a vibrationally excited state that is thermalized in about $9.5 \pm 0.5 \mathrm{ps}$. The positive and negative absorption recorded at the nanosecond time scale at $1631 \mathrm{~cm}^{-1}$ and $1961 \mathrm{~cm}^{-1}$ is the fingerprint of the MS1 photo-induced linkage isomer. This isomer has been shown to relax in 2270 ns [3]. As shown in Figure 3, these time-resolved measurements make it possible to picture the electronic and vibration evolution during formation of photo-induced linkage isomers in this crystal.

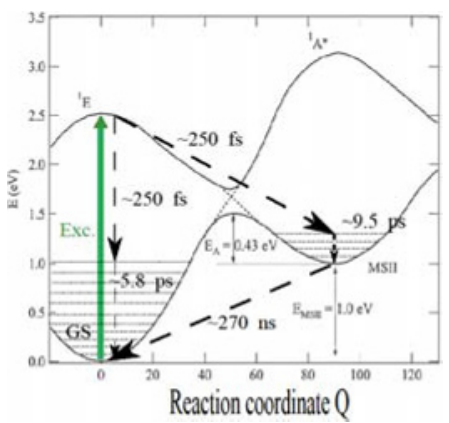

Fig. 3: Vibrationnal kinetic of the of SNP single crystals after a pulsed excitation centered at $500 \mathrm{~nm}$

\section{References}

[1] A. H. Zewail, J. Phys. Chem. 97, 12427 (1993)

[2] F. C. de Schryver, S. de Feyter, G. Schweitzer, Femtochemistry, (Wiley-VCH, Weinheim 2001).

[3] D. Schaniel, M. Nicoul, Th. Woike, Phys. Chem. Chem. Phys. 12, 9029 (2010).

[4] M. D. Carducci, M. R. Pressprich, P. Coppens, J. Am. Chem. Soc. 119, 2669 (1997).

[5] D. Schaniel, Th. Woike, J. Schefer, V. Petricek, Phys. Rev. B 71, 174112 (2005).

[6] D. Schaniel, Th. Woike, J. Schefer, V. Petricek, K. W. Krämer, H. U. Güdel, Phys. Rev. B 73, 174108 (2006). 